\title{
Ascending Aortic Constriction Promotes Cardiomyocyte Proliferation in Neonatal Rats
}

\author{
Zhenhua WANG, ${ }^{1}$ MD, Sebastian Schmull,${ }^{2} \mathrm{PhD}$, Hui Zheng,,${ }^{1} \mathrm{MD}$, \\ Jianggui Shan, ${ }^{1} \mathrm{MD}$, Rongjiang Zou, ${ }^{1} \mathrm{MD}$, and Song Xue, ${ }^{1} \mathrm{MD}$
}

\begin{abstract}
SUMMARY
Adult heart suffering from increased workload will undergo myocardial hypertrophy, subsequent cardiomyocyte (CM) death, and eventually heart failure. However, the effect of increasing afterload on the neonatal heart remains unknown. We performed ascending aortic constriction (AAC) in neonatal rats 8-12 hours after birth (P0, P indicates postpartum). Seven days after surgery, in vivo heart function was evaluated using cardiac ultrasonography. Haematoxylineosin and Masson staining were used to assess CM diameter and collagen deposition. Moreover, expression of both EdU and Ki67 were evaluated to determine DNA synthesis levels, and pH3 and aurora B as markers for mitosis in CMs. CM isolation was performed by heart perfusion at P0, P3, P5, and P7, respectively. CM number on P0 was $1.01 \pm 0.29 \times 10^{6}$. We found that $\mathrm{CM}$ cell cycle activation was significantly increased among constricted hearts, as demonstrated by increased Ki67, EdU, pH3, and aurora B positive cells/1000 CMs. At day 7 (P7), constriction group hearts manifested increased wall thickness $(0.55 \pm 0.05 \mathrm{~mm}$ versus $0.85 \pm 0.10 \mathrm{~mm}, P<0.01, n=6)$, and improved hemodynamics as well as left ventricular ejection fraction $(65.5 \pm 3.7 \%$ versus $77.7 \pm 4.8 \%, P<0.01, n=6)$. Of note, the population of CMs was also markedly increased in the constriction group $\left(2.92 \pm 0.27 \times 10^{6}\right.$ versus $\left.3.41 \pm 0.40 \times 10^{6}, P<0.05, n=6\right)$. In summary, we found that during the first week after birth significant numbers of neonatal CMs can reenter the cell cycle. Ascending aortic constriction promotes neonatal rat CM proliferation resulting in 16.7\% more CMs in the heart. (Int Heart J 2017; 58: 264-270)
\end{abstract}

Key words: Cell cycle reentry, Regeneration

$\mathrm{H}$ eart failure is an increasing burden on health care systems around the world. ${ }^{1)}$ Many forms of heart diseases result in significant and permanent losses of functional cardiomyocytes (CMs), such as coronary artery disease and pressure-loading cardiomyopathies. Efforts to address this issue have led to the exploration of methods for CM regeneration even though the mammalian heart has long been considered a postmitotic organ. Recently, it was shown that individual CMs may be not as old as the person - almost $50 \%$ of CMs are replaced during a normal life span. ${ }^{2)}$ By combining different genetic fate-mapping approaches, scientists have found that the genesis of CMs occurs by division of preexisting CMs. ${ }^{3,4)} \mathrm{Al}-$ though it happens at a very low rate, this evidence suggests that the heart is not terminally differentiated but instead an organ with regenerative potential. More lately, the discovery of an epimorphic regeneration potential in neonatal mammal hearts has contributed greatly to new insights in cardiac biology. In particular, neonatal mice can fully regenerate their heart after apex resection in a very short time. ${ }^{5)} \mathrm{BrdU}, \mathrm{pH} 3$ labeling, and genetic fate-mapping have indicated that the majority of regenerated $\mathrm{CMs}$ originated from preexisting ones. A similar conclusion was obtained by another research group working on a different animal model although they indicated that heart regeneration in neonatal mice was supported by c-kit ${ }^{+}$precursors. ${ }^{6}$ Moreover, it was demonstrated that even 2 weeks after birth CMs still can proliferate substantially in response to thyroid hormone. ${ }^{7)}$

\section{Editorial p.155}

Neonatal mouse heart retains significant regenerative potential during postnatal development. Whether the neonatal CMs can also re-enter the cell cycle and proliferate in response to pressure overload is still an unsolved question. The adult heart responds to pressure overload with significant hypertrophy and remodeling. ${ }^{8}$ However, this may not be the case for neonatal heart. A previous study indicated that neonatal rat heart regenerates following apical resection in a manner similar to that of neonatal mouse hearts. ${ }^{9)}$ We hypothesized that neonatal rat heart will also respond with $\mathrm{CM}$ proliferation to pressure overload. In this study, ascending aortic constriction (AAC) in neonatal rats was used to serve as a model of pressure overload. To the best of our knowledge, this is the first

From the ${ }^{1}$ Department of Cardiovascular Surgery, Ren Ji Hospital, School of Medicine, Shanghai Jiao Tong University and ${ }^{2}$ Ren Ji Med-X Clinical Stem Cell Research Center, Ren Ji Hospital, School of Medicine, Shanghai Jiao Tong University, Shanghai, P.R. China.

This work was supported by the Ren Ji hospital Zhong Zi Foundation (RJZZ13-023).

Address for correspondence: Song Xue, MD, Department of Cardiovascular Surgery, Ren Ji Hospital, School of Medicine, Shanghai Jiao Tong University, Building 7,

4th floor, Pujian Road 160\#, Shanghai 200127, P.R. China. E-mail renjixuesong@ sina.com

Received for publication May 15, 2016. Revised and accepted July 13, 2016.

Released in advance online on J-STAGE January 11, 2017.

All rights reserved by the International Heart Journal Association. 


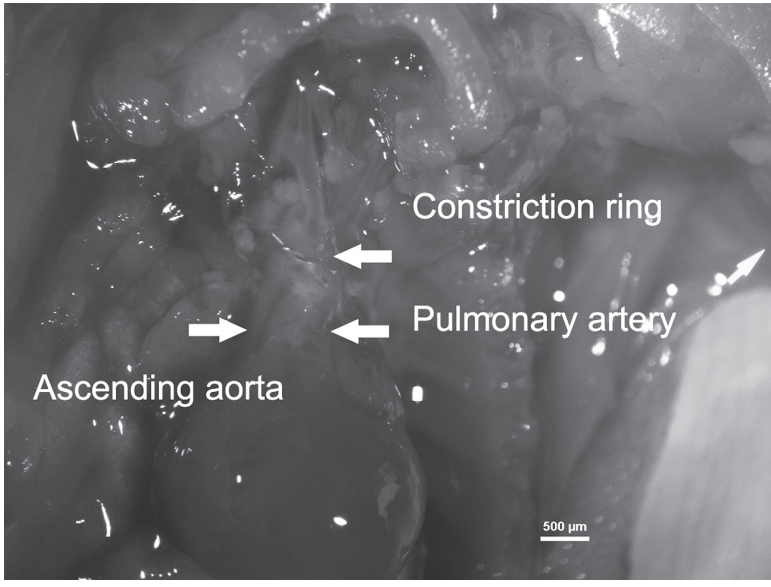

Figure 1. Intra-operative picture of ascending aortic constriction.

time an AAC model was established in neonatal rats. ${ }^{10)} \mathrm{We}$ constricted neonatal rat ascending aorta 8-12 hours after birth (P0), and found that the neonatal heart responded to such a pathological stimulus with massive $\mathrm{CM}$ proliferation during the following 7 days, resulting in a $16.7 \%$ increase in CMs.

\section{Methods}

Animals: Male or female neonatal rats from litters of 8-9 were used. All animals received care in compliance with the National Institutes of Health Guide for the Care and Use of Laboratory Animals (NIH Pub. No. 85-23, Revised 1996). This work was reviewed and approved by the Institutional Animal Care and Use Committee of Ren Ji Hospital (permit number 15RJ-0405). Neonates were anesthetized by cooling on ice for 5 minutes. Hearts were harvested while the animals were still under anesthesia. All efforts were made to minimize suffering.

Aortic constriction surgery: Newborn (day 0) rats (P0) were anesthetized by cooling on ice for 5 minutes. The thoracic cavity was then exposed by sternal interruption at the second intercostal space by blunt dissection. The ascending aorta was exposed by pulling the thymus laterally, isolated, and a copper wire ligature was tied around it $(0.55 \mathrm{~mm}$ constriction). Following aortic constriction, the thoracic wall incision and skin wound were sutured with 7-0 non-absorbable prolene suture (Figure 1). Rat pups were then brought back to body temperature by warming on a heated blanket. The entire procedure lasted about 13 minutes. Sham operation group animals were subjected to the same procedure without application of constriction rings.

Heart to body weight ratios: Seven days after surgery (P7), rat pups were weighed and, after excising, the hearts were rinsed with phosphate buffered saline (PBS). Afterwards, the hearts were pruned and drained by gently squeezing on absorbent paper before weighing.

Echocardiography: Transthoracic echocardiography was performed 7 days following AAC using a Visual Sonics Vevo 2100 echocardiograph (Toronto, Ontario, Canada) equipped with a $40 \mathrm{MHz}$ mouse ultrasound probe. Left ventricle function was calculated and posterior wall thickness was measured on conscious rats.

EdU pulse-chase: An Invitrogen Click-iT EdU Alexa 488 Imaging kit (Carlsbad, CA) was used for EdU detection. Rat pups were injected i.p. with $25 \mu \mathrm{g}$ EdU 1 day after surgery. Seven days after surgery, the hearts were harvested. After CM isolation, the cells were transferred to slides using cytospin, and fixed in $4 \%$ paraformaldehyde (PFA) at room temperature (RT) for 20 minutes. EdU detection was performed according to standard protocol.

Immunohistochemistry: Hearts were fixed in 4\% PFA at RT for 24 hours before paraffin embedding. Serial sections were cut at $5-\mu \mathrm{m}$ thickness through the entire heart, deparaffinized with xylene, and rehydrated. Hematoxylin/eosin and Masson trichrome staining was performed according to standard procedures.

CM isolation and dimension analyses: Rats were administered heparin sodium (10,000 U/kg i.p.) 20 minutes before anesthetization at different time points as mentioned above. The aorta was cannulated on a $0.64 \mathrm{~mm}$ needle and mounted on a Langendorff apparatus. Perfusion was initiated with buffer A (see end of section $)+$ EDTA $(0.2 \mathrm{mg} / \mathrm{mL}$, GIBCO, Paisley, UK) at 0.6-0.8 $\mathrm{mL} / \mathrm{min}$. A constant temperature of $37.5^{\circ} \mathrm{C}$ was maintained and the solution was gassed with $95 \% \mathrm{O}_{2}$ and $5 \% \mathrm{CO}_{2}$. After 5 minutes, buffer B was added to the perfusate for enzymatic dissociation of the tissue. Depending on heart size, digestion time varied from 9-14 minutes. Following digestion, both ventricles were obtained and cut in small pieces in solution A. After repeated pipetting, the resulting single cell solutions were immediately loaded in the counting chamber and counted in triplicate using a hemocytometer. For CM purification, single cell solutions were centrifuged for 5 minutes at 20 g. Images were acquired on an Olympus DP73 microscope (Tokyo). CM dimensions were measured using CellSens Standard software. CM length was determined by measuring the maximum Feret diameter, and width was evaluated by measuring the length of a line drawn perpendicular to the cell long-axis at the level of the nucleus. Buffer A contained (mM) $\mathrm{NaCl} 120, \mathrm{KCl} 15, \mathrm{KH}_{2} \mathrm{PO}_{4}$ 0.5, HEPES 10, $\mathrm{NaHCO}_{3} 5$, glucose 5 (pH 7.4, adjusted with $\mathrm{NaOH}$ ), and buffer B was composed of 190 units/mL collagenase II (Life Technologies, Carlsbad, CA) in Hank's Balanced Salt Solution with calcium and magnesium.

Immunofluorescence: Cells were prepared on cytospin slides $\left(2 \times 10^{5}\right.$ cells/slide $)$. Subsequently, specimens were fixed in $2 \%$ PFA for 15 minutes and permeabilized using $0.5 \%$ Triton $\mathrm{X}-100$ at room temperature for 20 minutes. Afterwards, samples were incubated overnight at $4{ }^{\circ} \mathrm{C}$ with the following primary antibodies: $\alpha$-sarcomeric actin (Abcam, Cambridge, UK), Ki67 (BD Biosciences, Bedford, MA), pH3 (Cell Signaling, Beverly, MA), and aurora B (Sigma, St. Louis, MO). The next day, samples were incubated for 60 minutes at RT with corresponding fluorochrome-conjugated secondary antibodies (Alexa Fluor 488 or 594 donkey anti-mouse, or anti-rabbit depending on primary antibody). All secondary antibodies were purchased from Life Technologies. DAPI was used to stain nuclei. Images were obtained with an Olympus DP73 microscope using Cellsens software. All CMs on a slide were counted and $\mathrm{Ki} 67$, pH3, or aurora B positive CMs were counted for calculation of percentages.

Statistical analysis: Statistical analyses were performed using a Prism software package (GraphPad version 4, San Diego, 
CA). Multiple group comparisons were performed by one-way analysis of variance (ANOVA) followed by the Bonferroni procedure for comparison of means. Comparisons between two groups were performed using the unpaired two-sided Student's $t$-test. All values are expressed as the mean \pm SEM. Differences were considered statistically significant when $P<$ 0.05 .

\section{RESUltS}

Mortality due to surgery and animal development: In general, sham or constriction operation caused less than $5 \%$ mortality. Aortic constriction severely affected development of experimental animals, and constriction group pups were generally $17.5 \%$ lighter in body weight. We did not sacrifice neonates at more than 8 days after constriction.

Aortic constriction induced cardiomegaly: Cardiomegaly was observed in rats after 1 week of transverse aortic constriction (Figure 2A-C). Of note, no significant fibrosis among the constricted hearts was present (Figure 2D-E). However, the inner dimension of the ascending aorta increased dramatically during the first week in the control group. In contrast, constriction surgery reduced the dimension of the ascending aorta $17 \%$ at $\mathrm{P} 0$, and the inner dimension of the ascending aorta remained less than $550 \mu \mathrm{m}$ thereafter (Figure $2 \mathrm{~F}$ ). To investigate the time-course of adaptation to constriction, we removed hearts at $1,3,5$, and 7 days after surgery. Compared with the sham operated controls, aortic constricted pups were associated with decreased gain of body weight. On the contrary, heart weight was markedly increased in the constriction group and the heart weight-to-body weight ratio also showed a significant increase (Figure 2G). To assess the development of cardiomegaly, morphologic and echocardiographic analyses were performed. Left ventricle interior diastolic diameter (LVIDd) and left ventricle interior systolic diameter (LVIDs) decreased 7 days after constriction. LV wall thickness values were significantly higher in the constriction group and the left ventricular ejection fraction was also increased, suggesting strengthened systolic performance (Figure 2H).

Cell cycle reentering of CMs after aortic constriction: As stated earlier, we sacrificed rat pups at different time points after surgery. Subsequently, the hearts were digested and CMs were isolated. Staining for $\alpha$-sarcomeric actin and proliferation marker Ki67 showed that marked numbers of CMs were in interphase in the sham-operated hearts. The fraction of Ki67 positive CMs among constricted hearts was $25.6 \pm 0.2 \%$ in comparison to $16.2 \pm 1.8 \%$ in the sham operation group $(P<$ $0.05, n=5$ ) (Figure 3A, B). Furthermore, we injected neonatal rats with a single intraperitoneal dose of EdU 24 hours after surgery. Labeled CM nuclei comprised $\sim 4.5 \%$ o in the sham-operated group, and $\sim 10 \%$ in the constriction group (Figure 3C, D), indicating that more CMs entered $\mathrm{S}$ phase after constriction $(P<0.05, n=5)$. Staining of $\mathrm{pH} 3$ confirmed further that a vast number of CMs were reentering cell cycle after constriction $(4 \pm 0.1 \%$ versus $24.5 \pm 9.3 \%$ o $P<0.05, n=5$ ) (Figure $3 \mathrm{E}, \mathrm{F})$. We next identified mitotic CMs by evaluating the coexpression of aurora $\mathrm{B}$ and $\alpha$-sarcomeric actin. A significantly higher number of aurora $\mathrm{B}$ positive $\mathrm{CMs}$ was found in constricted hearts $(\sim 5.6 \%)$ relative to sham-operated hearts $(\sim 0.9 \%, P<0.05, n=5)$ (Figure 4A, B). Thus, neonatal rat
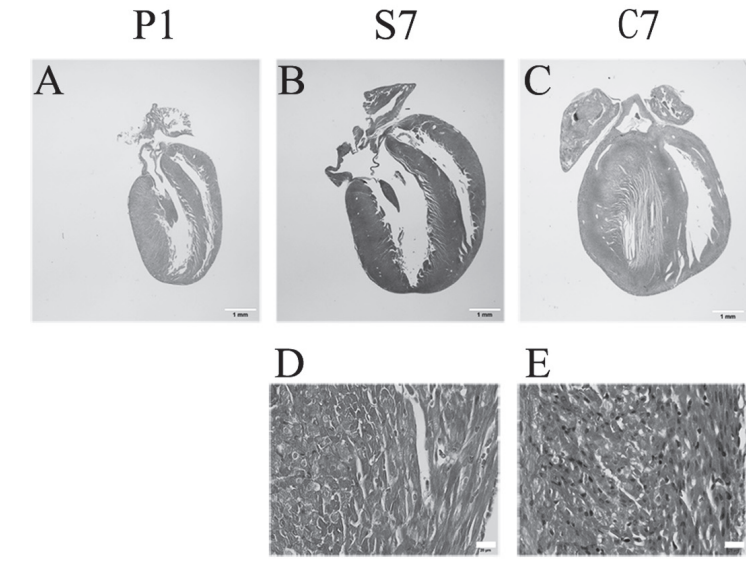

F

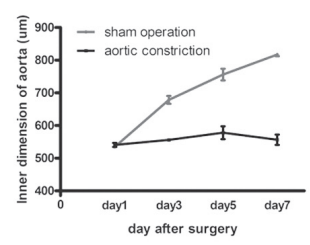

$\mathrm{H}$
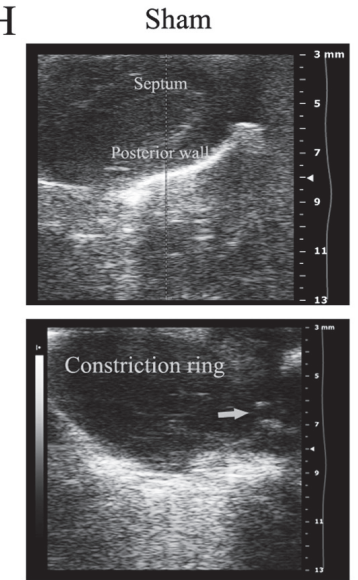

Figure 2. Cardiomegaly was induced in constricted hearts. A: Hematoxylin and eosin (HE) staining of neonatal rat heart at P1 (day 1 postpartum). B, C: Masson's trichrome staining of rat heart 7 days after surgery. Significant cardiac enlargement was induced in C7 (7 days after AAC) compared to S7 (7 days after sham operation). Scale bars $1 \mathrm{~mm}$. Masson's trichrome staining showed no significant increase of extracellular collagen at 7th day after constriction, S7 (D) compared with C7 (E). Scale bars 200 $\mu \mathrm{m}$. F: The inner dimension of ascending aorta increased from 534.3 \pm 5.4 $\mu \mathrm{m}$ to $836 \pm 31.1 \mu \mathrm{m}$ in the first week after birth. In contrast, constriction surgery reduced the dimension of ascending aorta by $17 \%$ at P0, and the inner dimension of the ascending aorta remained less than $550 \mu \mathrm{m}$ thereafter. (G) Heart and body weight ratio increased 24 hours after constriction $(P=0.027, n=5)$, and remained at a high level in the constriction group. Values are presented as the mean \pm SEM; ${ }^{*} P<0.05,{ }^{* * *} P<0.001$. H: Images of echocardiogram in the long axis view of sham and constriction surgery. Heart function was strengthened and the left ventricle wall was thickened by constriction surgery. Arrow denotes ascending aortic constriction site. Lower panel shows left ventricular (LV) dimensions and function of hearts 7 days after surgery. Left ventricular internal diameter at end systole (LVIDs) and end diastole (LVIDd) were used to calculate ejection fraction $(\% \mathrm{EF})$. The thickness of the left ventricle is indicated by the posterior wall thickness (PWT) and interventricular septum thickness (IVST). Values are presented as the mean \pm SEM; $n=5$ in each group. 
A
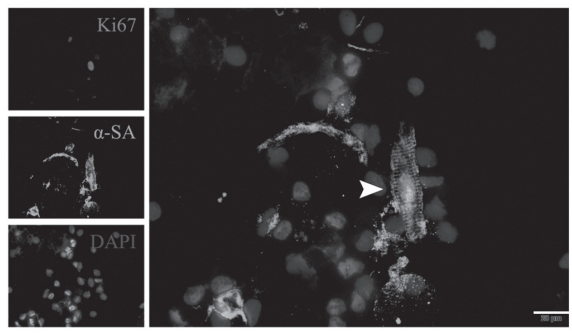

$\mathrm{C}$
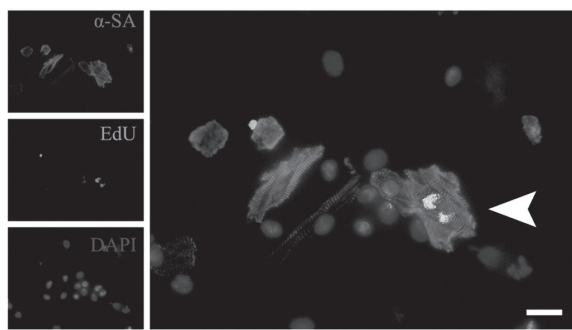

E
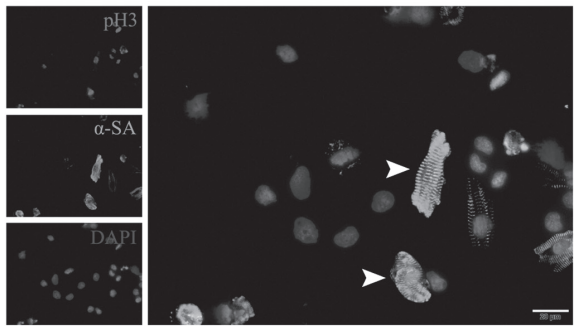

B
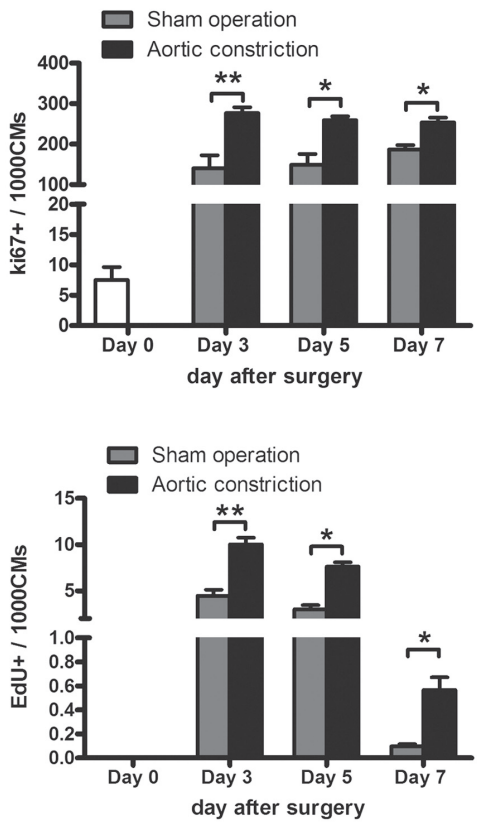

F

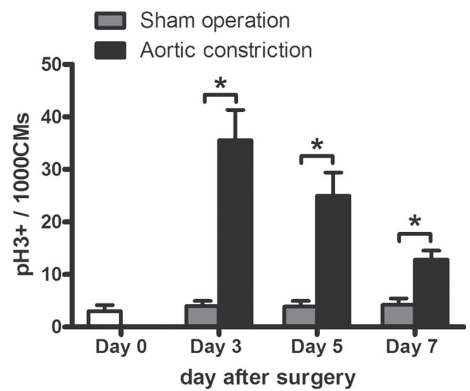

Figure 3. AAC increases cell cycle reentry in neonatal rat heart. A, C and E: Representative images of Ki67+ (red), EdU+ (green), and pH3+(red) CMs. Arrowheads denote CMs positive for Ki67, EdU or pH3, respectively. Scale bars $20 \mu \mathrm{m}$. B, D and F: Quantification of Ki67+, EdU+ and pH3+ / $1000 \mathrm{CMs}$ at different time points after surgery. Significant amounts of $\mathrm{Ki} 67+\mathrm{EdU}+$ or $\mathrm{pH} 3+\mathrm{CMs}$ were present in the first week after AAC. Values are presented as the mean $\pm \mathrm{SEM} ;{ }^{*} P<0.05$, ${ }^{* *} P<0.01$.

hearts exhibited strong molecular markers expression of cell cycle progression after constriction.

Cardiomyocytes were not hypertrophic and their number increased after AAC: Cardiomegaly induced by constriction is possibly the consequence of an increase in cardiac myocyte size. Therefore, we measured the size of myocytes after CM isolation from P0, P3, P5, and P7 rat hearts. More than 100 CMs from each time point were evaluated (Figure 4C). CM size continues to increase during the first week after birth, but the increase in $\mathrm{CM}$ width or length among constricted hearts compared to sham-operated hearts was not statistically different (Figure 4D, E). As the next step, we determined total CM numbers in ventricles by enzymatic disaggregation and direct cell counting at different time points as above. Neonates were divided into 3 groups; no surgery, sham operation, and constriction groups. CM number on P0 (surgery day) was $1.01 \pm$ $0.29 \times 10^{6}(n=6)$. After 7 days, cell numbers were $2.85 \pm 0.28$ $\times 10^{6}$ during normal development (no surgery) and $2.92 \pm 0.27$ $\times 10^{6}$ after sham operation $(n=6)$. In the constriction group, the $\mathrm{CM}$ number was $3.41 \pm 0.40 \times 10^{6}$, which was significantly larger than the no surgery group or sham operation group $(P<$ $0.05, n=6$ ) (Figure $4 \mathrm{~F}$ ).

\section{Discussion}

In our study, we provided direct evidence that there is a brief time window for neonatal rats in which remarkable CM proliferation happens. During normal development, neonatal rat $\mathrm{CM}$ population almost tripled in the first week after birth. By stimulation of transverse ascending aortic constriction, neonatal CM numbers increased by $\sim 16.7 \%$ in the heart. Indeed, based on our results, we found that the neonatal heart responds to increased afterload with a significantly increased CM popu- 
A

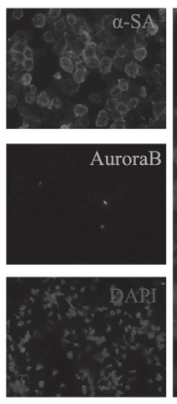

C

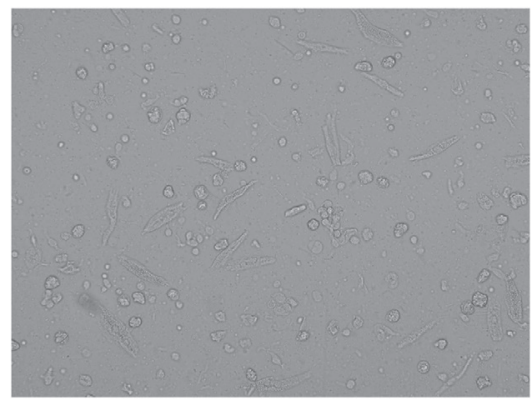

E

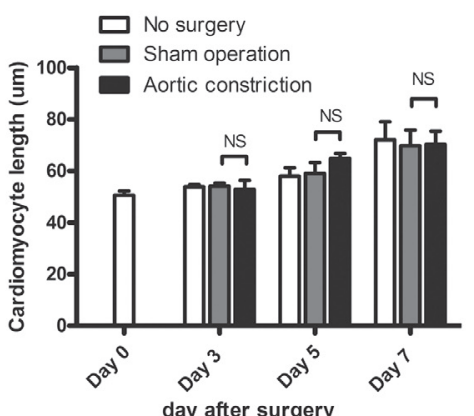

B

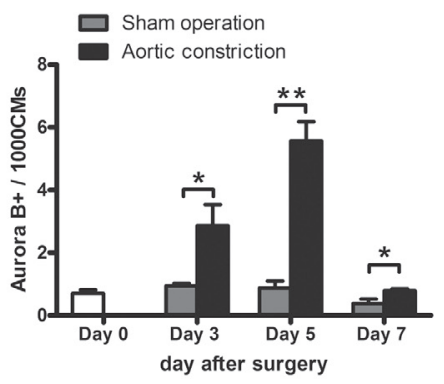

$\mathrm{D}$

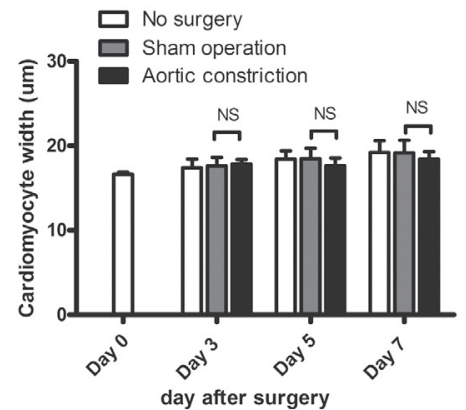

F

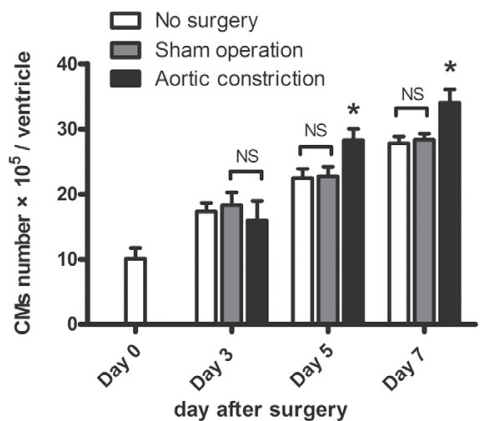

Figure 4. Large amounts of CMs were added to neonatal hearts, and the proliferative burst involves division of CMs. A: Identification of mitotic CMs by aurora B (green) staining. Arrowheads denote a cytokinetic CM with aurora B kinase staining. Scale bars $20 \mu \mathrm{m}$. B: Quantitation of aurora B+ CMs. Quantification represents counts per $1000 \mathrm{CMs}$ from 3 independent samples per group. C: CMs just isolated from cardiac ventricles. CMs are large, rod-shaped, and easily identifiable for counting. D, E: Histograms represent average length and width of 7-day-old neonatal rat CMs ( $n=50-70 \mathrm{CMs}$ /heart from 4-6 rats per group). There were no significant differences between groups. F: Number of CMs in ventricles of 0, 3, 5, and 7-day-old rats. Population of CMs was significantly larger 5 days after aortic constriction. Values are presented as the mean \pm SEM; ${ }^{*} P<0.05$.

lation and cell cycle reentry activities, which is different to the adult heart.

Myocardial infarction and heart failure are global concerns. Coronary heart disease alone accounts for 1 of every 7 deaths in the United States. ${ }^{11)}$ Strategies to improve the body's natural repair and regeneration potential seem to be a theoretically ideal therapy. But adult cardiac myocytes are terminally differentiated cells which have lost the ability to proliferate. Learning from neonates about how to stimulate CM proliferation is a potentially good strategy. In this study, we showed that various amounts of $\mathrm{CMs}$ were strongly expressing $\mathrm{Ki} 67$, $\mathrm{pH} 3$, and aurora B during the early days after constriction. Moreover, sarcomere disassembly accompanied this process. This observation is consistent with mouse-specific results and implies that dedifferentiation and proliferation maybe the main cause for regeneration. ${ }^{5)}$ After the period of P3-P5, we observed that the ability of CM proliferation decreased dramatically and was lowest on the 7th day. Of note, expression of the cytokinesis-related genes $\mathrm{pH} 3$ and aurora $\mathrm{B}$ also falls precipitously. Therefore, the first week after birth is a very important 
time window for $\mathrm{CM}$ s to proliferate. However, more attention should be focused on P3 to P5 since during this time period the expression of $\mathrm{pH} 3$ and $\mathrm{Ki} 67$ as well as EdU labeling is strongest, indicating active CM proliferation. Although we found large amounts of CMs were generated after AAC surgery in neonatal rats, this does not necessarily imply that the underlying mechanism is similar to a ventricle apex resection model or myocardial infarction model. There are substantial differences between the heart injury model and aortic banding model, not only marked differences in the inflammatory response to injury, but also differences in the requirement for re-vascularization and angiogenesis following injury. A recent study showed that macrophages provide necessary signals to drive angiogenesis and $\mathrm{CM}$ regeneration. ${ }^{12)}$ It would be very interesting to determine if they are present in our constriction model and contribute to $\mathrm{CM}$ proliferation.

Hypertrophy, an increase in cell size, may be the only form of growth response available for adult CMs after disease or physical stimuli, such as long-standing hypertension, myocardial infarction, valvular disease, or physical activity. ${ }^{13)}$ In a typical pathological process, long term cardiac hypertrophy will remodel the heart, including the following mechanisms: fibrosis, electric remodeling, and cavity dilatation, predisposing individuals to heart failure. Indeed, studies have demonstrated that intense myocyte formation emerged during the process of hypertrophy. ${ }^{14,15)}$ In our study, we did not find significant hypertrophy of CMs, nor did we find notable fibrosis of the neonatal rat heart after AAC. Instead, we found that a large number of CMs were added to the neonatal heart, and echocardiography of 7 days old rats showed strengthened cardiac functions as compared to sham-operated rats. Consequently, we propose that the cell cycle machinery has not been entirely switched off in the first week postpartum. Thus, CMs underwent hypertrophy after we constricted again the ascending aorta of P7 neonatal rats. Interestingly, CMs were significantly larger in the constriction group compared to sham-operated rats as revealed by Masson's trichrome staining which was performed additional 7 days later (P14) (data not shown). To add further support to our hypothesis, this observation needs to be addressed in future experiments.

The first animal model of aortic constriction was developed by Goldblatt, et al. ${ }^{16)}$ By constriction of the aorta above the origin of both renal arteries, they produced a secondary form of hypertension in adults resulting in cardiac hypertrophy. After constriction, the elevation of blood pressure is not simultaneous, but develops in about 24-48 hours after constriction. Several weeks or months later, there is also a tendency for the elevated pressure to return to the normal level. Renal sympathetic nerve activity plays a pivotal regulatory role in this process and renal sympathetic denervation attenuates myocardial fibrosis and hypertrophy. ${ }^{17)}$ However, our model of ascending aortic constriction is different and necessary for the study of CM proliferation. First, AAC imposed the increased work load almost immediately on the heart, while the effect of abdominal aortic constriction was observable only $24-48$ hours later. As we have shown above, the first 3 days after birth is a critical time interval for CMs to proliferate. After this time period, the proliferation potential of neonatal rat heart diminishes very quickly. Thus, it is important to increase heart afterload before CMs exit the cell cycle. Second, the effect of abdominal aortic constriction is considered to be of kidney origin and its mecha- nism on cardiac hypertrophy is indeed different to our model, which is available for the adult heart. ${ }^{10)}$ Consequently, it would be interesting to compare the effect of abdominal aortic constriction and ascending aortic constriction on neonatal rat heart.

The physiological adaptation of the neonatal heart to increased work load may have different mechanisms compared to adult heart. ${ }^{18)}$ Our results showed that large amounts of new CMs were generated in this process. However, we did not investigate the activation of cardiac stem cell-like populations, such as c-kit ${ }^{+}, \mathrm{Scal}^{+}$cells.6, ${ }^{19-21)}$ Studies have indicated that neonatal-derived cardiac stem cells have a strong regenerative ability when compared with adult-derived cardiac stem cells. $^{22,23)}$ In our results, we also noticed that there are large numbers of CMs generated in the normal development of neonatal rat. Since we did not investigate the occurrence and frequency of cardiac stem cells, it is extremely difficult for us to rule out cardiac stem cells as the source of new CM formation. Thus, myocyte-restricted lineage tracing is needed for future work to address this issue.

The endogenous regenerative potentials of adult hearts are extremely limited and different regenerative procedures have been explored. ${ }^{24,25)}$ Here we show that neonatal rat CMs possess high proliferative potential and response with active proliferation of CMs to increased afterload. Thus, ascending aorta constriction in neonatal rats may provide an attractive method to explore efficient CM generation therapy for endstage heart disease.

\section{Disclosure}

None.

\section{REFERENCES}

1. McMurray JJ, Adamopoulos S, Anker SD, et al. ESC guidelines for the diagnosis and treatment of acute and chronic heart failure 2012: The Task Force for the Diagnosis and Treatment of Acute and Chronic Heart Failure 2012 of the European Society of Cardiology. Developed in collaboration with the Heart Failure Association (HFA) of the ESC. Eur J Heart Fail 2012; 14: 803-69.

2. Bergmann O, Bhardwaj RD, Bernard S, et al. Evidence for cardiomyocyte renewal in humans. Science 2009; 324: 98-102.

3. Ali SR, Hippenmeyer S, Saadat LV, Luo L, Weissman IL, Ardehali R. Existing cardiomyocytes generate cardiomyocytes at a low rate after birth in mice. Proc Natl Acad Sci U S A 2014; 111: 8850-5.

4. Senyo SE, Steinhauser ML, Pizzimenti CL, et al. Mammalian heart renewal by pre-existing cardiomyocytes. Nature 2013; 493: 433-6.

5. Porrello ER, Mahmoud AI, Simpson E, et al. Transient regenerative potential of the neonatal mouse heart. Science 2011; 331: 1078-80.

6. Jesty SA, Steffey MA, Lee FK, et al. c-kit+ precursors support postinfarction myogenesis in the neonatal, but not adult, heart. Proc Natl Acad Sci U S A 2012; 109: 13380-5.

7. Naqvi N, Li M, Calvert JW, et al. A proliferative burst during preadolescence establishes the final cardiomyocyte number. Cell 2014; 157: 795-807.

8. Boluyt MO, Robinson KG, Meredith AL, et al. Heart failure after long-term supravalvular aortic constriction in rats. Am J Hypertens 2005; 18: 202-12.

9. Zogbi C, Saturi de Carvalho AE, Nakamuta JS, et al. Early post- 
natal rat ventricle resection leads to long-term preserved cardiac function despite tissue hypoperfusion. Physiol Rep 2014; 2: e12115.

10. Molina EJ, Gupta D, Palma J, et al. Novel experimental model of pressure overload hypertrophy in rats. J Surg Res 2009; 153: 28794.

11. Mozaffarian D, Benjamin EJ, Go AS, et al. Heart disease and stroke statistics--2015 update: a report from the American Heart Association. Circulation 2015; 131: e29-322.

12. Aurora $\mathrm{AB}$, Porrello ER, Tan $\mathrm{W}$, et al. Macrophages are required for neonatal heart regeneration. J Clin Invest 2014; 124: 1382-92.

13. Radovits T, Oláh A, Lux Á, et al. Rat model of exercise-induced cardiac hypertrophy: hemodynamic characterization using left ventricular pressure-volume analysis. Am J Physiol Heart Circ Physiol 2013; 305: H124-34.

14. Urbanek K, Quaini F, Tasca G, et al. Intense myocyte formation from cardiac stem cells in human cardiac hypertrophy. Proc Natl Acad Sci U S A 2003; 100: 10440-5.

15. Waring CD, Vicinanza C, Papalamprou A, et al. The adult heart responds to increased workload with physiologic hypertrophy, cardiac stem cell activation, and new myocyte formation. Eur Heart J 2014; 35: 2722-31.

16. Goldblatt H, Kahn JR, Hanzal RF. Studies on experimental hypertension: IX. The effect on blood pressure of constriction of the abdominal aorta above and below the site of origin of both main renal arteries. J Exp Med 1939; 69: 649-74.

17. Li ZZ, Jiang H, Chen D, et al. Renal sympathetic denervation im- proves cardiac dysfunction in rats with chronic pressure overload. Physiol Res 2015; 64: 653-62.

18. Sedmera D, Thompson RP, Kolar F. Effect of increased pressure loading on heart growth in neonatal rats. J Mol Cell Cardiol 2003; 35: 301-9.

19. Ellison GM, Vicinanza C, Smith AJ, et al. Adult c-kit(pos) cardiac stem cells are necessary and sufficient for functional cardiac regeneration and repair. Cell 2013; 154: 827-42.

20. Uchida S, De Gaspari P, Kostin S, et al. Sca1-derived cells are a source of myocardial renewal in the murine adult heart. Stem Cell Reports 2013; 1: 397-410.

21. Holmes C, Stanford WL. Concise review: stem cell antigen-1: expression, function, and enigma. Stem Cells 2007; 25: 1339-47. (Review)

22. Simpson DL, Mishra R, Sharma S, Goh SK, Deshmukh S, Kaushal S. A strong regenerative ability of cardiac stem cells derived from neonatal hearts. Circulation 2012; 126: S46-53.

23. Zaruba MM, Soonpaa M, Reuter S, Field LJ. Cardiomyogenic potential of C-kit(+)-expressing cells derived from neonatal and adult mouse hearts. Circulation 2010; 121: 1992-2000.

24. Yamakawa H, Ieda M. Strategies for heart regeneration: approaches ranging from induced pluripotent stem cells to direct cardiac reprogramming. Int Heart J 2015; 56: 1-5. (Review)

25. Hashimoto A, Naito AT, Lee JK, et al. Generation of induced pluripotent stem cells from patients with Duchenne muscular dystrophy and their induction to cardiomyocytes. Int Heart J 2016; 57: 112-7. 